\title{
Synthesis of Ladder-type Radio Frequency Surface Acoustic Wave Filter Based on Lumped Circuit Model by Using Neural Network
}

\author{
Mei-Hui Chung, ${ }^{1}$ Huang-Chu Huang, ${ }^{2}$ Rey-Chue Hwang, ${ }^{3}$ \\ Pei-Fen Huang, ${ }^{3}$ and Shuming T. Wang ${ }^{3 *}$ \\ ${ }^{1}$ Office of Institutional Research, I-Shou University, \\ No. 1, Sec. 1, Syuecheng Rd., Dashu District, Kaohsiung City 84001,Taiwan, R.O.C. \\ ${ }^{2}$ Department of Electronic Communication Engineering, National Kaohsiung University of Science and Technology, \\ No. 142, Haijhuan Rd., Nanzih District, Kaohsiung City 81157, Taiwan, R.O.C. \\ ${ }^{3}$ Department of Electrical Engineering, I-Shou University, \\ No. 1, Sec. 1, Syuecheng Rd., Dashu District, Kaohsiung City 84001,Taiwan, R.O.C.
}

(Received November 29, 2018; accepted March 7, 2019)

Keywords: SAW resonator, ladder-type SAW filter, neural network

With the development of mobile communication technology, the demand for mobile devices continues to rise. Acoustic devices such as radio frequency (RF) filters and duplexers are widely used in mobile handsets because of their excellent electric performance characteristics. However, the key bottleneck is how to convert a lumped circuit model into the structure parameters of an acoustic resonator for RF circuit designers so that they can apply RF circuit design tools in designing surface acoustic wave (SAW) devices. The neural network (NN) has been widely applied in various science and management areas for its powerful learning and mapping capabilities. In this paper, a method of building a bridge between the lumped equivalent circuit (EC) model and structure parameters of a SAW resonator is proposed. The approach begins with establishing a database that shows the relationship between the values of the lumped EC and corresponding structure parameters of the SAW resonator. A four-layered $\mathrm{NN}$ with the back-propagation (BP) algorithm is adopted to determine the relationship between the structure parameters of the SAW resonator and the lumped EC. Then, other input/output pairs are presented to check if the estimated lumped EC matched with original circuit. Finally, as an example, the design of a long term evolution (LTE) transmitter (Tx) filter is developed to demonstrate the proposed approach.

\section{Introduction}

Acoustic devices such as radio frequency (RF) filters and duplexers are widely used in mobile handsets because of their excellent electric performance characteristics such as their compactness, low insertion loss, and high out-of-band isolation. In general, there are two main RF surface acoustic wave (SAW) filter configurations according to the connection type for mobile phone applications, namely, ladder-type and double-mode SAW (DMS) filters. ${ }^{(1-9)}$

*Corresponding author: e-mail: smwang@isu.edu.tw

https://doi.org/10.18494/SAM.2019.2208 
The ladder-type filter, which consists of several resonators cascaded in series and parallel, is more commonly applied to an RF front-end filter because of its high selectivity. ${ }^{(2,5,6,9)}$ With the growth of the 4th generation of mobile wireless broadband communication transmission technology [4G, long term evolution (LTE)], the latest-generation smartphones are expected to support most of the frequency bands and require more filters in a device. However, increasingly complex specifications, such as extremely high rejection of adjacent frequency bands with low insertion loss, and time-to-market pressure have reinforced the need for advanced filter design techniques. In addition, because of the smaller footprints and excellent frequency performance characteristics of RF SAW filters, the trend now is to integrate multiple filters into RF modules with switches and power amplifiers. ${ }^{(10-12)}$

Figure 1 shows the lumped circuit model of a one-port acoustic resonator near resonance, where $L_{m}$ is the motional inductance, $C_{m}$ is the motional capacitance, $R_{m}$ is the motional resistance, and $C_{T}$ is the static capacitance. ${ }^{(5,6)}$ Although well-experienced RF circuit designers can understand the behavior of the components in the network by using a related high-frequency computer-aid software program, the key bottleneck is how to convert the lumped circuit model into structure parameters, which include the period of the interdigital transducer (IDT) $(\lambda)$, the number of IDT pairs $\left(N_{p}\right)$, the IDT aperture $(A)$, the metal thickness ratio of the electrode $(h / \lambda)$, and the metallization ratio of the SAW resonator $(\eta)$.

In 1943, McCulloch and Pitts reported a model of a neuron that shows key features of biological neurons. ${ }^{(13)}$ Since the proposal of the back-propagation (BP) algorithm by Rumelhart and coworkers in $1986,{ }^{(14,15)}$ the neural network (NN) has been widely applied in various science and management areas. ${ }^{(16-19)}$ Because of the powerful learning and mapping capabilities of $\mathrm{NN}$, the complex and nonlinear relationship between input and output parameter pairs can be obtained easily through a simple training process.

In this paper, an NN that builds a bridge between the lumped equivalent circuit (EC) model and the structure parameters of a SAW resonator is proposed. The approach begins with establishing the database of the values of the lumped EC corresponding to the structure parameters of the SAW resonator. A four-layered NN with the BP learning algorithm is adopted to determine the relationship between the structure parameters of the SAW resonator and the lumped EC. Then, other input/output pairs are presented to test the estimation accuracy. Finally, a procedure of designing an RF ladder-type SAW filter is developed to demonstrate the proposed approach, which will effectively shorten the development time of ladder-type SAW filters.

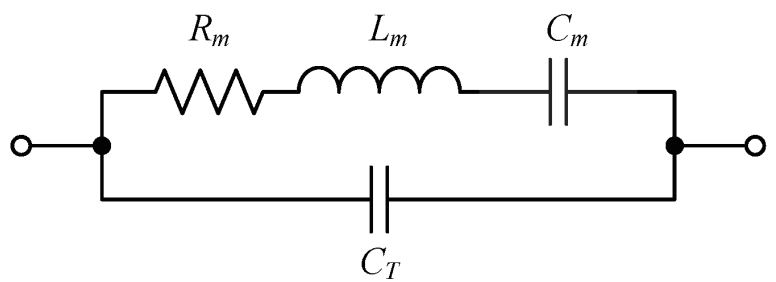

Fig. 1. Lumped circuit model of a one-port acoustic resonator. 


\section{Method}

\subsection{Electrical performance of acoustic resonators}

Typical impedance characteristics of a one-port SAW resonator are illustrated in Fig. 2 indicating two resonances, namely, the resonance frequency (or series frequency, $\omega_{r}$ ), where the magnitude of impedance tends to be minimum $\left(Z_{r}\right)$, and the antiresonance frequency (or parallel frequency, $\left.\omega_{a}\right)$, where the magnitude of impedance tends to be maximum $\left(Z_{a}\right)$. They are determined from piezoelectric substrate material parameters, which include wave velocity, electromechanical coupling coefficient, attenuation constant, dielectric constant, and so forth, and structure parameters of the SAW resonator, such as the lengths of the IDT and reflector electrodes, electrode metal thickness, and electrode overlap. The components of the lumped circuit model shown in Fig. 1 can be derived from the properties of the resonator. ${ }^{(6)}$

$$
\begin{gathered}
\omega_{r}=\frac{1}{\sqrt{L_{m} C_{m}}} \\
\omega_{a}=\frac{1}{\sqrt{L_{m} C_{m} C_{T} /\left(C_{m}+C_{T}\right)}}
\end{gathered}
$$

$R_{m}$ can be derived from the conductance $\left(G^{-1}\right)$ at the resonance frequency and $C_{T}$ represents the IDT capacitance. The capacitance ratio $(\gamma)$ is an important factor used to evaluate the resonator performance and is given by ${ }^{(6)}$

$$
\gamma=\frac{C_{T}}{C_{m}}=\frac{1}{\left(\omega_{a} / \omega_{r}\right)^{2}-1} .
$$

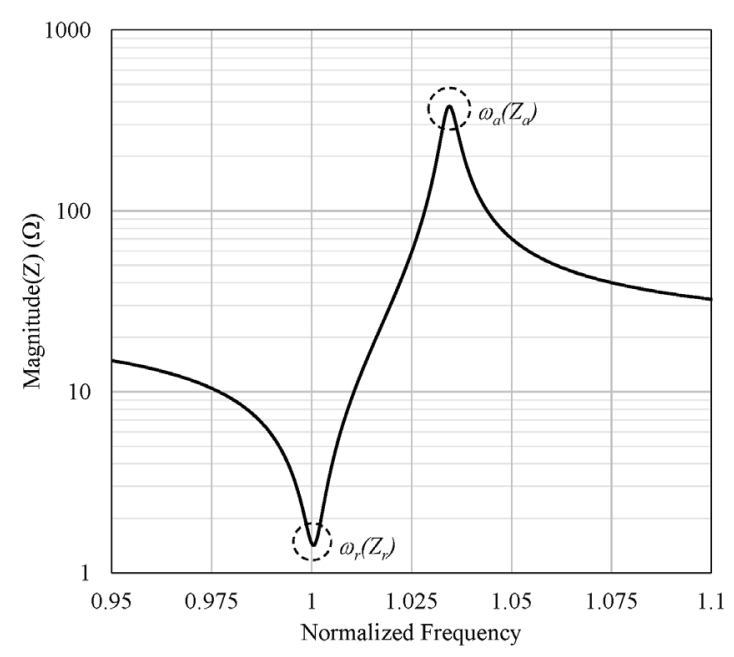

Fig. 2. Impedance characteristics of a one-port SAW resonator. 
The quality factor $\left(Q_{r}\right)$ at the resonance frequency is also an important measure and is expressed as $^{(6)}$

$$
Q_{r}=\omega_{r} L_{m} / R_{m}=1 / \omega_{r} C_{m} R_{m}
$$

To establish a reliable database for an NN to determine the relationship between the lumped and structure parameters of the SAW resonator, the accuracy of predicting the performance of a SAW resonator is crucial. In this work, a new modified EC method that converts couplingof-mode (COM) parameters into EC parameters with a metallization ratio of 0.5 was applied to analyze the characteristics of the SAW resonator. The simulated and measured impedance characteristics of a one-port SAW resonator were examined and found to be consistent as shown in Fig. 3.

\subsection{Structure parameters of SAW resonator determined using NN}

The error BP learning algorithm is taken for NN's training to find the structure parameters of the SAW resonator from the known lumped EC model. The impedance characteristics of the SAW resonator simulated by using a new modified EC method and by calculating from the corresponding lumped EC were compared and verified, as shown in Fig. 4. Furthermore, a database that shows the correspondence between the structure parameters of the one-port SAW resonator and the components of the lumped EC was established by computing. Here, a fourlayered NN consisting of an input layer, an output layer, and two hidden layers was used as shown in Fig. 5. There are four input parameters, namely, the $f_{r}, C_{T}, \gamma$, and $Q_{r}$ of the lumped EC, and five output parameters, namely, $\lambda, N_{p}, A, h / \lambda$, and $\eta$ of the SAW resonator. The NN model

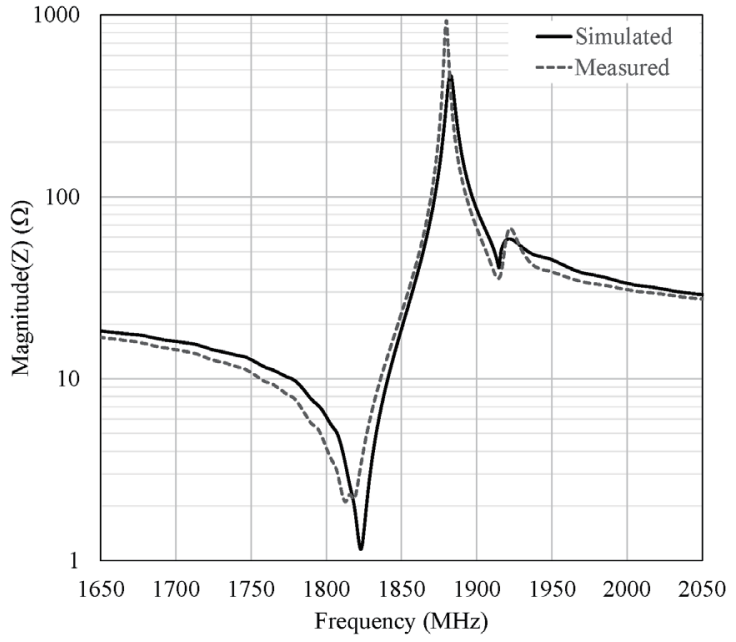

Fig. 3. Simulated and measured impedance characteristics of a one-port SAW resonator.

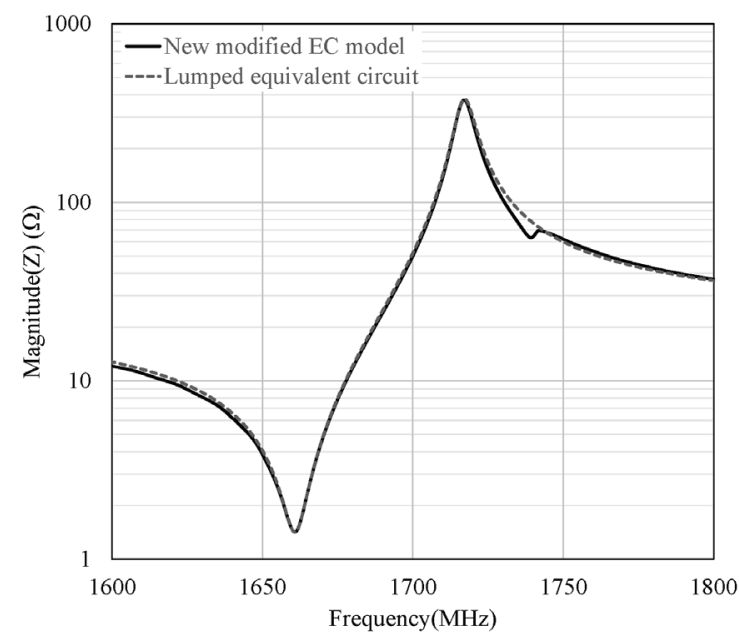

Fig. 4. Impedance characteristics of the SAW resonator simulated by using a new modified EC method and calculating from the corresponding lumped EC. 


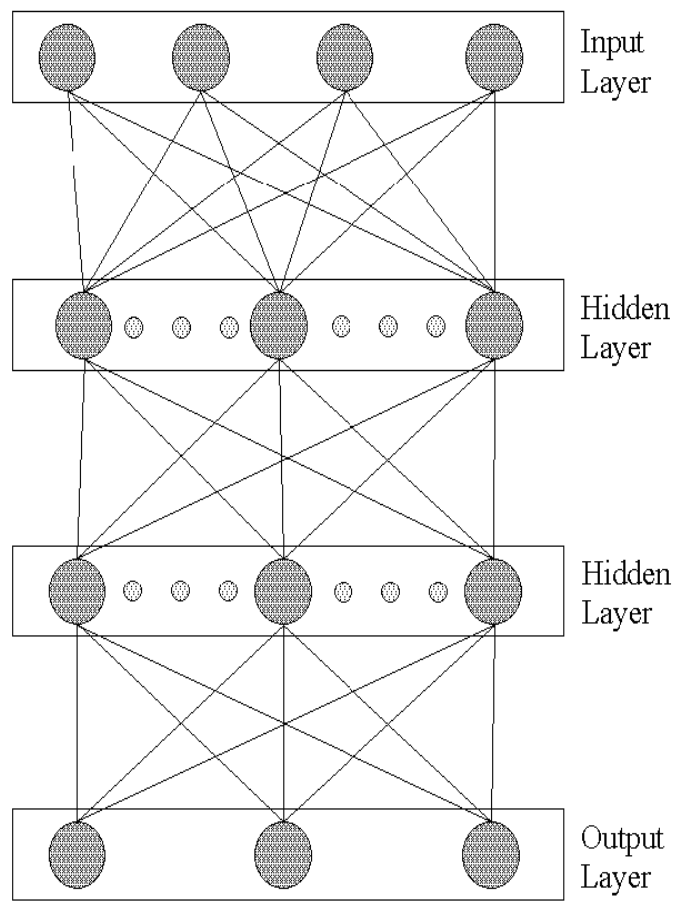

Fig. 5. Structure of four-layered NN.

was trained and determined by using the established database, and other input/output pairs are presented for testing and verifying the learning results.

\subsection{Synthesis ladder-type SAW filters using lumped element model}

Figure 6 shows a simple one-level ladder-type filter with one resonator in series and one in a shunt denoted by $\mathrm{UE}_{1}$ and $\mathrm{UE}_{2}$, respectively. Typically, in order to achieve a band pass response, the aim is to adjust parameters to set the resonant frequency of $\mathrm{UE}_{1}\left(\omega_{1 r}\right)$ to be nearly coincident with the antiresonant frequency of $\operatorname{UE}_{2}\left(\omega_{2 a}\right)$ as shown in Fig. . $^{(5)}$ The ratio of $C_{1 T}$ to $C_{2 T}$ determines both the insertion loss of the filter and its out-of-band rejection level. Note that the more the ladder stages, the better the out-of-band rejection, but the worse the pass band insertion loss. In a practical design, the designer may need a certain design trade-off. ${ }^{(5)}$ Hence, the procedure of synthesizing ladder-type SAW filters using the lumped element model is suggested as follows:

(a) Determine the ladder stages of the desired filter.

(b) Use RF circuit design software to optimize the lumped circuits and obtain the $L_{m}, C_{m}, R_{m}$, and $C_{T}$ of each resonator.

(c) Determine the structure parameters $\lambda, N_{p}, A, h / \lambda$, and $\eta$ of each SAW resonator by using an NN.

(d) Simulate and adjust the response of the filter. 


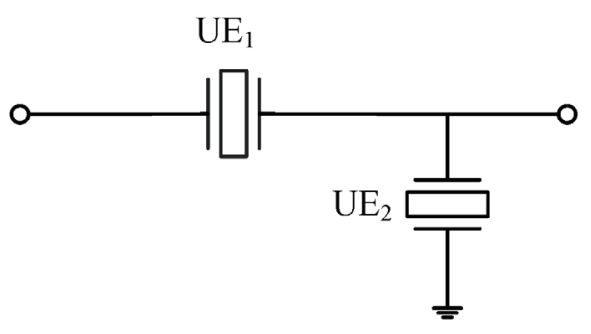

(a)

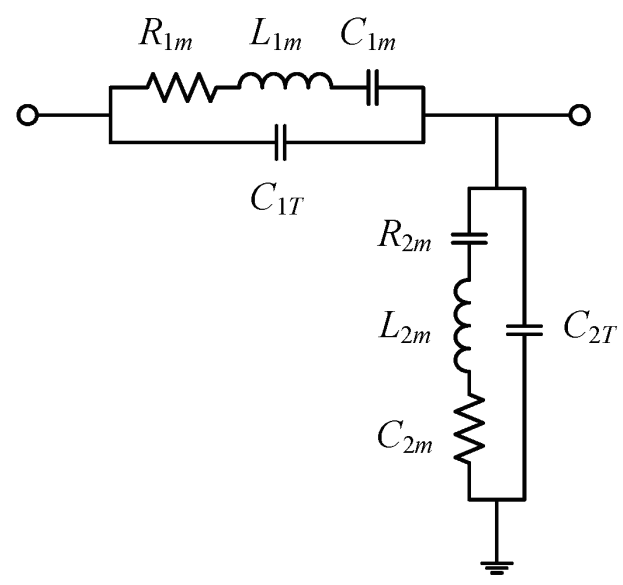

(b)

Fig. 6. (a) Simple ladder-type filter and (b) lumped EC.

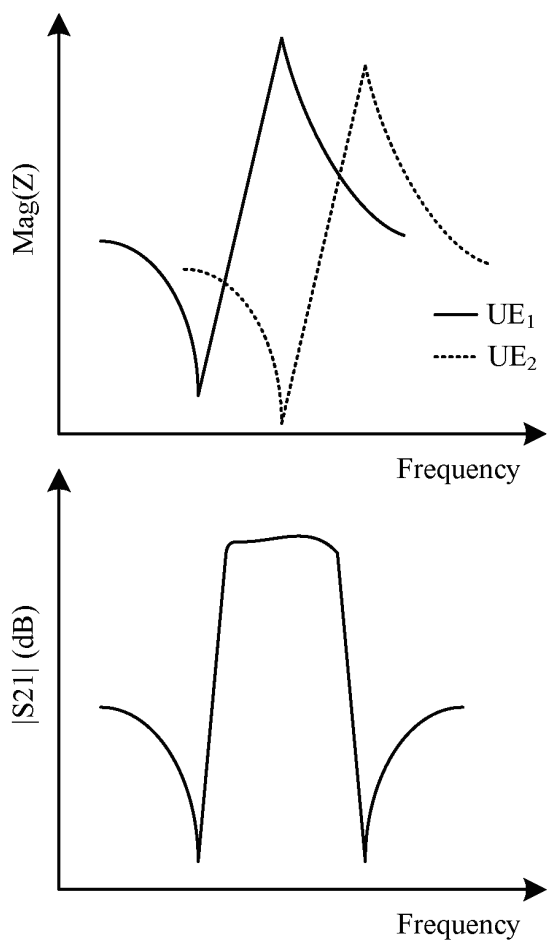

Fig. 7. Band pass characteristics of the ladder-type filter.

\section{Results and Discussion}

\subsection{Electrical performance of acoustic resonators}

The lithium tantalite $\left(\mathrm{LiTaO}_{3}\right)$ piezoelectric substrate, which is most commonly used in an RF SAW filter because of its high electromechanical coupling coefficient, was chosen in this work. The frequency was set to about $1670 \mathrm{MHz}$. The effects of structure parameters of the 
SAW resonator on the characteristics of this substrate were studied by simulation. The IDT static capacitance $C_{T}$ is ${ }^{(5,6)}$

$$
C_{T}=C_{0} N_{p} A,
$$

where $C_{0}$ is the capacitance per unit length of one IDT pair and its value is related to the characteristics of the piezoelectric substrate. $C_{T}$ is proportional to the product of $N_{p}$ and A. Equation (3) can also be used to describe the relationship between the resonance and antiresonance frequencies of the resonator. In Fig. $8, \gamma$ decreases with increasing $N_{p}$, indicating that the bandwidth of the resonator (defined as the difference between the resonance and antiresonance frequencies) increases with $N_{p}$. However, $\gamma$ tends to be flat owing to the limitation of the properties of the piezoelectric substrate.

Since the electrode metal thickness ratio $h / \lambda$ strongly affects the electromechanical coupling coefficient, the velocity under metal electrodes, and the reflection of IDTs and reflectors, it is an important factor for determining the resonance frequency and bandwidth of the SAW resonator. Figure 9(a) shows that $f_{r}$ decreases with increasing $h / \lambda$ owing to the surface loading. Also, the bandwidth of the resonator increases with $h / \lambda$, as shown in Fig. 9(b). The metallization ratio $\eta$ varies the static capacitance of $C_{T}$ and may also affect the resonance frequency and bandwidth. From the simulation results, $f_{r}$ is observed to greatly decrease and the bandwidth is determined to decrease with increasing $\eta$, as plotted in Fig. 10.

\subsection{SAW parameters determined using NN}

Considering the limitation of the manufacture and component size, the ranges of structure parameters of the SAW resonator for the database are listed in Table 1. The database of various SAW resonators with structure parameters within the ranges was simulated and its

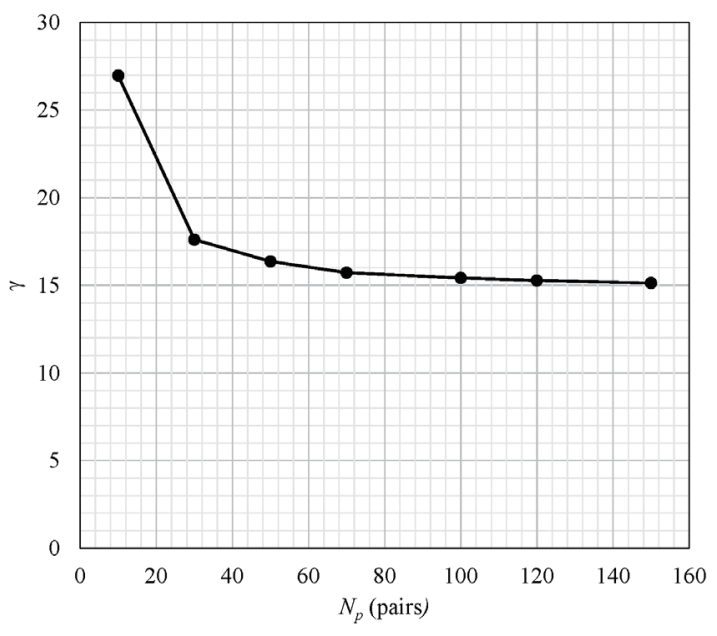

Fig. 8. $\gamma$ versus $N_{p}$. 


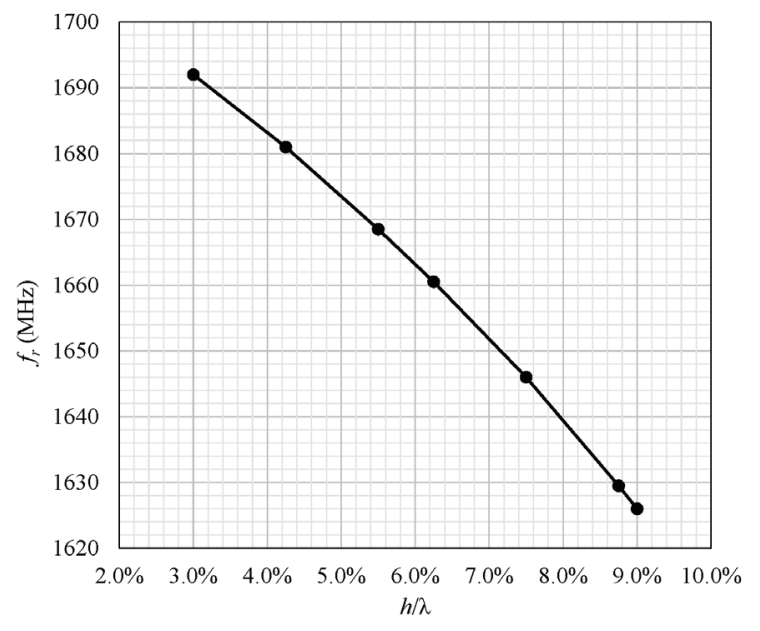

(a)

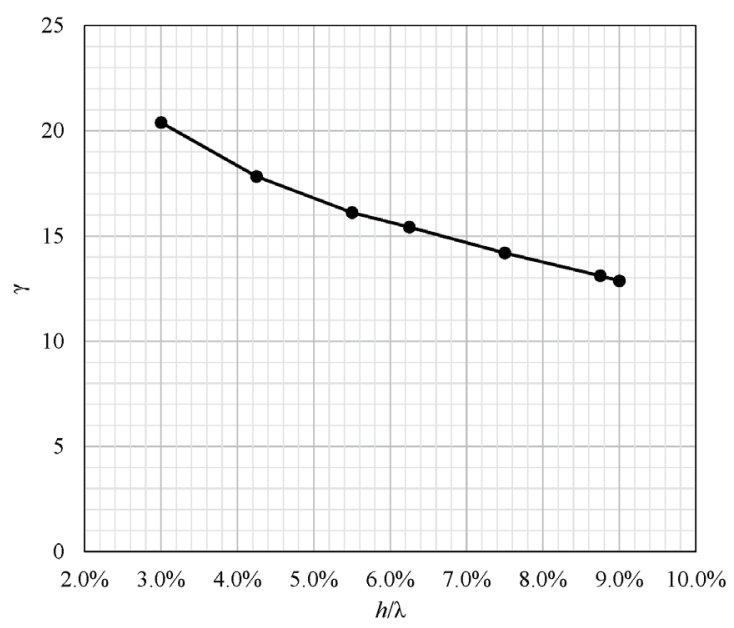

(b)

Fig. 9. Relationships of $h / \lambda$ with (a) $f_{r}$ and (b) $\gamma$.

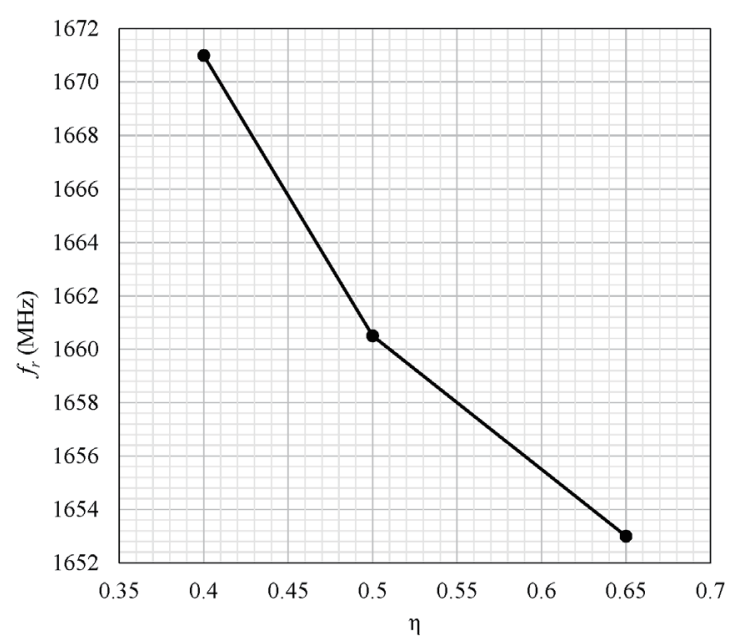

(a)

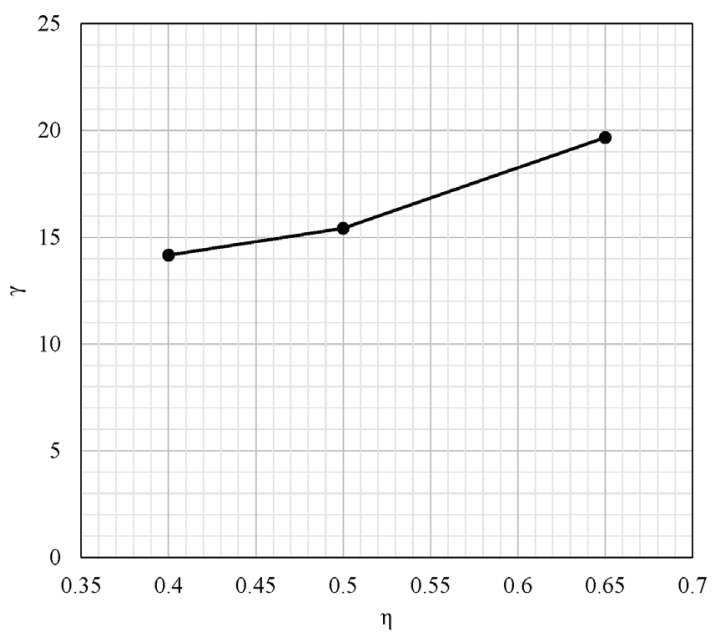

(b)

Fig. 10. Relationships of $\eta$ with (a) $f_{r}$ and (b) $\gamma$.

Table 1

Ranges of structure parameters of SAW resonator for building database.

\begin{tabular}{lccccc}
\hline Parameter & $\lambda$ & $N_{p}$ & $A$ & $h / \lambda$ & $\eta$ \\
\hline Range & $1.5-2.4 \mu \mathrm{m}$ & $10-150$ pairs & $5-70 \lambda$ & $3-9 \%$ & $0.4-0.65$ \\
\hline
\end{tabular}

corresponding lumped model was calculated. From the database, 882 sets of data were selected for NN's training and 50 sets of data were used for checking. Once the NN was well trained, another 45 sets of data were applied to the $\mathrm{NN}$ reversely, i.e., giving lumped parameters and obtaining the structure parameters of the SAW resonator to verify the accuracy of the NN method. As shown in Fig. 11, the frequency responses of the lumped circuit model and learned SAW were consistent except for the spurious mode because the lumped circuit model does not include the spurious effect. 


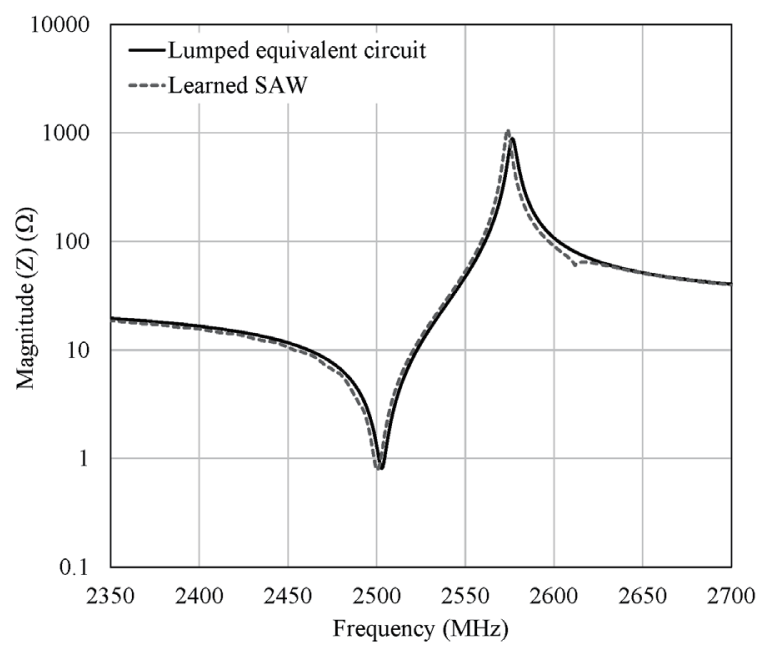

Fig. 11. Impedance characteristics of the lumped EC model and learned SAW.

Table 2

LTE Tx filter specifications.

\begin{tabular}{lcc}
\hline Response & $\begin{array}{c}\text { Frequency band } \\
(\mathrm{MHz})\end{array}$ & $\begin{array}{c}\text { Values } \\
(\mathrm{dB})\end{array}$ \\
\hline Insertion loss & $2500-1915$ & $<3.5$ \\
\hline \multirow{3}{*}{ Out-of-band rejection } & $2400-2472$ & $>20$ \\
& $2472-2480$ & $>10$ \\
& $2480-2484$ & $>4$ \\
& $2620-2690$ & $>15$ \\
\hline
\end{tabular}

Table 3

Synthesized components of the lumped circuit models of LTE Tx filter.

\begin{tabular}{lccc}
\hline Parameters & Res_S & Res_P1 & Res_P2 \\
\hline$f_{r}(\mathrm{MHz})$ & 2548.87 & 2452.18 & 2452.18 \\
$C_{T}(\mathrm{pF})$ & 0.5774 & 0.9568 & 2.1671 \\
$\gamma$ & 12.59 & 13.47 & 13.90 \\
$Q_{r}$ & 348 & 321 & 337 \\
\hline
\end{tabular}

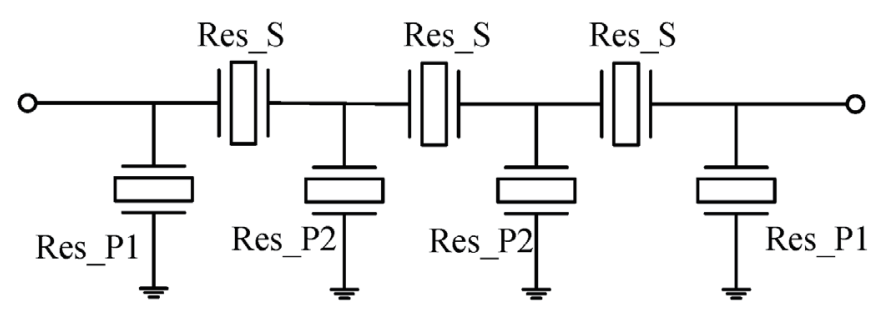

Fig. 12. Six-stage ladder structure.
Table 4

Structural parameters of the corresponding SAW resonators in Table 3.

\begin{tabular}{lccc}
\hline Parameters & Res_S & Res_P1 & Res_P2 \\
\hline$\lambda(\mu \mathrm{m})$ & 1.54 & 1.6 & 1.6 \\
$N p$ (pairs) & 114 & 76 & 150 \\
$A(\lambda)$ & 7.5 & 17 & 19.5 \\
$\eta$ & 0.48 & 0.5 & 0.52 \\
$h / \lambda(\%)$ & $\sim 9$ & $\sim 8.3$ & $\sim 8.3$ \\
\hline
\end{tabular}

\subsection{Ladder-type SAW filters using synthesized lumped element model}

In this section, an example of an LTE transmitter (Tx) filter, whose specifications are shown in Table 2, was designed to demonstrate the proposed approach. It is a six-stage ladder structure, as shown in Fig. 12. The components of the lumped circuit models of each resonator were determined by using computer-aid circuit design software and their values are listed in Table 3. The corresponding structure parameters of each SAW resonator obtained by using a well-trained NN model are presented in Table 4. The frequency responses $\left(\mathrm{S}_{21}\right)$ of the synthesized ladder-type SAW filter with the learned SAW structure and the original lumped circuit are shown in Fig. 13. The results show good agrement. 


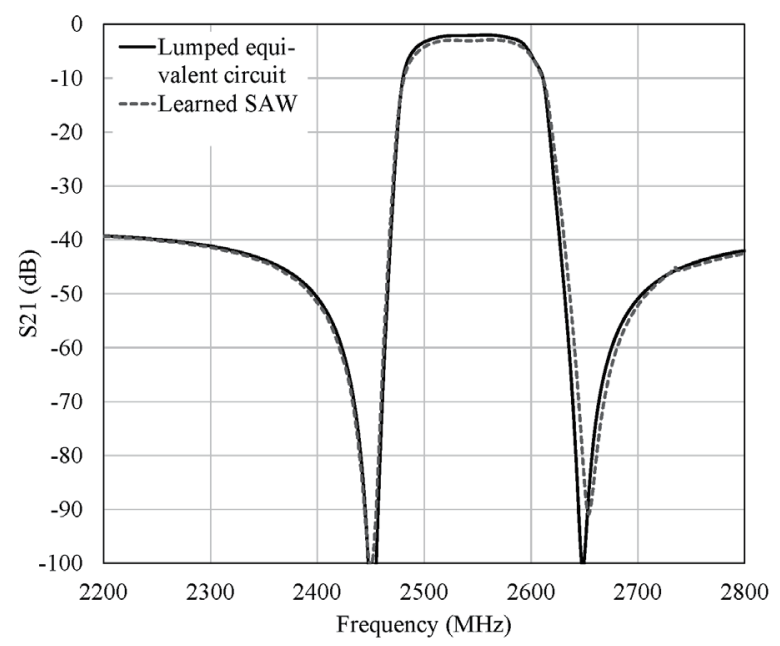

Fig. 13. Frequency responses (S21) of the synthesized ladder-type SAW filter. The solid line shows the response for the learned SAW and the dotted line shows the response for the lumped EC model.

\section{Conclusions}

In this study, a bridge between the lumped EC model and structure parameters of a SAW resonator was successfully established by using an NN. A four-layered NN with the BP learning algorithm was adopted to obtain the structure parameters of the SAW resonator corresponding to the lumped EC. Results were consistent except for the spurious effect. To demonstrate the advantage of using the proposed method in designing a ladder-type SAW filter, an LTE Tx filter was developed. It can be seen that the proposed method effectively shortens the time for developing SAW filters.

\section{Acknowledgments}

The authors thank the Ministry of Science and Technology, Taiwan, for partially supporting this research under Contract No. MOST 106-2221-E-214-003.

\section{References}

1 T. Morita, Y. Watanabe, M. Tanaka, and Y. Nakazawa: Proc. IEEE 1992 Ultrason. Symp. (IEEE, 1992$) 95$. https://doi.org/10.1109/ULTSYM.1992.276057

2 O. Ikata, T. Miyashita, T. Matsuda, T. Nishihara, and Y. Satoh: Proc.1992 Ultrason. Symp. (IEEE, 1992) 110. https://doi.org/10.1109/ULTSYM.1992.276055

3 N. Kimura, M. Nakano, M. Nakazawa, and K. Sato: Jpn. J. Appl. Phys. 36 (1997) 3101. http://iopscience.iop. org/article/10.1143/JJAP.36.3101/meta

4 H. H. Ou, N. Inose, and N. Sakamoto: Proc. 1998 IEEE Ultrason. Symp. (IEEE, 1998) 97. https://doi. org/10.1109/ULTSYM.1998.762107

5 C. K. Campbell: Surface Acoustic Wave Devices for Mobile and Wireless Communications (Academic Press, Inc., San Diego/Toronto, 1998) p. 286.

6 K. Hashimoto: Surface Acoustic Wave Devices in Telecommunications, Modelling and Simulation (Springer Verlag, Berlin/New York, 2000) p. 123.

7 C. C. W. Ruppel, L. Reindl, and R. Weigel: IEEE Microwave Mag. 3 (2002) 65. https://doi.org/10.1109/ MMW.2002.1004053 
8 F. M. Pitschi, J. E. Kiwitt, R. D. Koch, B. Bader, K. Wagner, and R. Weigel: Proc. 2009 IEEE Ultrason. Symp. (IEEE, 2009) 1. https://doi.org/10.1109/ULTSYM.2009.5441550

9 T. Komatsu, Y. Tanaka, K. Hashimoto, T. Omori, and M. Yamaguchi: IEEE Trans. Ultrason. Ferroelectr. Freq. Control 56 (2009) 1451. https://doi.org/10.1109/TUFFC.2009.1200

10 J. Min, Z. Wu, M. R. Pulugurtha, V. Smet, V. Sundaram, A. Ravindran, and C. Hoffmann: Proc. 2016 IEEE 66th Electronic Components and Technology Conf. (ECTC) (IEEE, 2016) 1297. https://doi.org/10.1109/ ECTC.2016.310

11 K. Matsutani, M. Tani, H. Tsukamoto, A. Horita, K. Onaka, T. Wada, M. Koshino, M. Kawashima, and N. Nakajima: Proc. 2017 IEEE MTT-S Int. Microwave Symp. (IMS) (IEEE, 2017) 1679-1682. https://doi. org/10.1109/MWSYM.2017.8058963

12 B. v. Liempd, A. Visweswaran, S. Ariumi, S. Hitomi, P. Wambacq, and J. Craninckx: IEEE Trans. Microwave Theory and Tech. 65 (2017) 4621. https://doi.org/10.1109/TMTT.2017.2728039

13 W. S. Mcculloch and W. H. Pitts: Bull. Math. Biophys. 5 (1943) 115. https://link.springer.com/article/10.1007/ BF02478259

14 D. E. Rumelhart, G. E. Hinton, and R. J. Williams: Nature 323 (1986) 533. https://www.nature.com/ articles $/ 323533 \mathrm{a} 0$

15 D. R. Rumelhart and J. L. Mcclelland: Parallel Distributed Processing: Explorations in the Microstructure of Cognition (Foundations, United States, 1986) p. 283.

16 J. Dheeba, N. A. Singh, and S. T. Selvi: J. Biomed. Inf. 49 (2014) 45. https://doi.org/10.1016/j.jbi.2014.01.010

17 Y. Goldberg: J. Artif. Intell. Res. 57 (2016) 345. https://doi.org/10.1613/jair.4992

18 M. A. RafeBiswas, M. D. Robinson, and N. Fumo: Energy 117 (2016) 84. https://doi.org/10.1016/ j.energy.2016.10.066

19 B. Shi, X. Bai, and C. Yao: IEEE Trans. Pattern Anal. Mach. Intell. 39 (2017) 2298. https://doi.org/10.1109/ TPAMI.2016.2646371

\section{About the Authors}

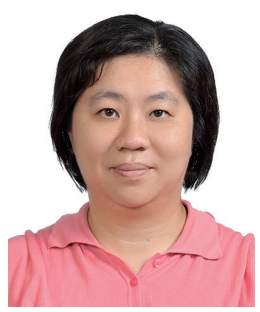

Mei-Hui Chung received her M.S. and Ph.D. degrees in electrical engineering from I-Shou University, Taiwan, in 2000 and 2007, respectively. She currently serves as a section chief of Institutional Research at I-Shou University, Taiwan. Her research interests are in surface acoustic wave device design and high-frequency circuit design. (mhchung@isu.edu.tw)

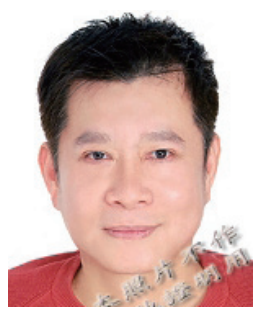

Huang-Chu Huang received his Ph.D. degree in electrical engineering from National Sun Yat-Sen University, Taiwan, in year 2001. Currently, he is a professor of the Electronic Communication Department, National Kaohsiung University of Science and Technology, Kaohsiung City, Taiwan. His research interests are in the areas of control, power signal prediction, and neural network applications. (h4530@nkust.edu.tw)

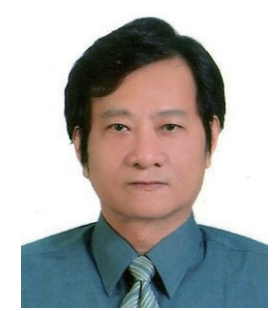

Rey-Chue Hwang received his Ph.D. degree in electrical engineering from Southern Methodist University, Dallas, TX, in 1993. Currently, he is a full professor of the Electrical Engineering Department, I-Shou University, Taiwan, R.O.C. Dr. Hwang has published more than 250 papers in various journals and conferences in the areas of artificial intelligent system, signal processing, and fuzzy control. He is now a fellow of IET and a senior member of IEEE. He chartered the IEEE CIS Chapter, Tainan Section and served as the co-chair and chair from 2004 to 2009. (rchwang@isu.edu.tw) 
Pei-Fen Huang received her B.S. degree in electrical engineering from I-Shou University in 2017 and is currently in the M.S. program of electrical engineering, I-Shou University. (gcobs064046@yahoo.com.tw)

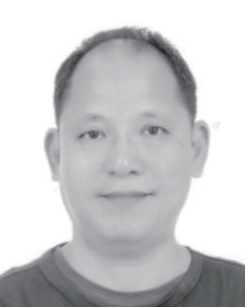

Shuming T. Wang received his B.S. degree from National Chiao Tung University, Taiwan, in 1983 and his M.S. and Ph.D. degrees from Marquette University, USA, in 1986 and 1992, respectively. Since 1993, he has been an associate professor of I-Shou University, Taiwan. His research interests are in RF circuit design and acoustic wave devices. (smwang@isu.edu.tw) 